\title{
Failure of additivity in bisection of length
}

\author{
NORMAN H. ANDERSON \\ University of California, San Diego, La Jolla, California 92093
}

\begin{abstract}
Subjects were intructed to select one rod to lie halfway in length between two given rods. These bisection instructions imply an additive model in the subjective metric. However, the data were inherently nonadditive; the length of the bisector could be an increasing or a decreasing function of the length of one given rod, depending on the length of the other given rod. A convexity analysis and a nonmetric analysis both showed that no monotone transformation could make the data additive. The bisection problem is used to contrast the axiomatic and functional approaches to measurement theory.
\end{abstract}

Bisection has long been popular in psychophysics, mainly because of its relation to Fechner's problem of determining the psychophysical law. The bisection task is attractive because it requires only direct sensory comparisons and measures the stimuli on their observable physical metric. There is no need to rely on arbitrary verbal responses, or on uncertain assumptions that all jnds are equal.

Unfortunately, the bisection problem has long resisted satisfactory analysis (see Fagot, 1961; Marks, 1974; Weiss, 1973, 1975). Many treatments have assumed no order effects, for example, contrary to results for loudness and brightness (Gage, 1934). Other treatments have simply assumed the validity of some model without testing and validating it. Thus, the equisection analysis used in the mel scale for pitch rests squarely on the untested assumption that the equisection model is valid (Weiss, 1973). As Fagot remarks, "The significance of psychophysical laws based on psychological scales that have not been validated is dubious at best"' $(1961$, p. 127).

Functional measurement has provided a new approach to bisection. The essential idea "consists in using the postulated behavior laws to induce a scaling on the dependent variable" (Anderson, 1962). Theoretical and experimental applications of functional measurement to the problem of bisection have been given by Anderson (1970, 1974a, 1976b), Bogartz and Wackwitz (1971), and Weiss (1973, 1975).

In principle, the analysis of bisection data is simple. Bisection is expected to obey an averaging

This work was supported by National Science Foundation Grant BMS 74-19124, and by grants from the National Institute of Mental Health to the Center for Human Information Processing, University of California, San Diego. Thanks are due Carlyn Joergensen and Cliff Butzin for running these experiments, and to Cliff Butzin and Jim Zalinski for assistance in data analysis. Author's address: Psychology, C-009, University of California, La Jolla, California 92093. model: The subjective value of the bisector stimulus should be an average of the subjective values of the two bisectee stimuli. Thus, the subjective value of the response will be additive, showing a pattern of parallelism in a factorial design.

The observable response, of course, is on the physical scale and so will not generally be additive itself. However, it can be assumed that subjective value is some strictly monotone function of physical value. If the bisection model is true, therefore, then some monotone transformation will make the observed data additive. Indeed, this transformation is the psychophysical law itself.

In principle, therefore, bisection analysis involves only two problems: First, a way to compute the needed monotone transformation; second, a test of goodness of fit. In practice, these two problems have presented considerable difficulty but straightforward methods are now available.

This functional measurement approach has been successfully applied to validate the bisection model for grayness (Anderson, 1976b; Weiss, 1975). For length, which is studied in this article, the bisection model fails. This failure is of interest, for it shows that the model cannot be taken for granted, as has been done in constructing the mel scale for pitch. The failure is also of interest for its illustration of a new error theory for nonmetric analysis.

\section{BISECTION AND MULTISECTION MODELS}

The statement of the bisection model is slightly complicated by the need to take account of both subjective and physical values. The symbols $S$ and $s$ are used to denote the physical and the subjective values of the stimulus. The function $V$ relating them,

$$
\mathrm{V}(\mathrm{S})=\mathrm{s},
$$

is the psychophysical function or law. Since $S$ is known, the determination of $\mathrm{V}$ is equivalent to 
measurement of subjective value, and vice versa.

Let $S_{1}$ and $S_{2}$ be the two stimuli to be bisected, with subscripts referring to temporal or spatial position. Let $\mathbf{R}$ denote the physical value of the bisector response, with $r$ the corresponding subjective value. Then $V(R)=r$, and $R=V^{-1}(r)$. It is assumed that $V$ is strictly monotone so that $\mathrm{V}^{-1}$ is uniquely defined.

A common conceptualization of bisection is that the subject is equating sense differences. In terms of the unobservable subjective metric, this conceptualization can be written:

$$
w_{1}\left(r-s_{1}\right)=w_{2}\left(s_{2}-r\right),
$$

where the weights allow for differential attention to the two sense distances.

If Equation 2 is solved for the subjective response, the bisection model takes the form of an averaging model:

$$
r=\left(w_{1} s_{1}+w_{2} s_{2}\right) /\left(w_{1}+w_{2}\right) .
$$

Unless otherwise stated, it is assumed that $w_{1}+w_{2}$ $=1$, so the denominator in Equation 3 can be ignored.

This bisection model can also be written in terms of the observable physical metric by use of the $\mathrm{V}$ function and its inverse. Since $R=V^{-1}(r)$, Equation 3 becomes

$$
R=V^{-1}\left[w_{1} V\left(S_{1}\right)+w_{2} V\left(S_{2}\right)\right]
$$

Equation 4 has two essential properties. First, the quantity inside the brackets is an additive function of the two stimulus variables. Second, $\mathrm{V}^{-1}$ is strictly monotone, being the inverse of the strictly monotone function $V$. Thus, $R$ is a monotone transformation of an additive function, so that some monotone transformation will make $R$ additive. Further, since $\mathrm{VV}^{-1}$ is the identity function, Equation 4 shows that the required transformation is just the psychophysical law V.

If the bisection model is true, therefore, then the observed data can be monotonically transformed to additivity. All that is required for this analysis is the observable response matrix of physical values, R. No other assumptions are necessary, either about the form of the psychophysical law, about the subjective values, or about the weight parameters.

The weight parameters deserve special mention. They allow for spatial position effects and for temporal order effects. The so-called "hysteresis" effect, for example, may be merely a temporal order effect. If these effects are present, of course, then the bisection is not at the midpoint between the two stimuli so that, in model terms, unequal weights would be required. However, the analysis handles unequal weights automatically.
The weight parameter thus allows for generalizations of the common bisection task. For example, the subject could be instructed to section at the quarterpoint or at any arbitrary proportion. Consistent use of one proportion is all that is necessary. Similarly, the model could be applied directly to multisection in which the subject chooses several stimuli to cut up the interval between the two given stimuli into several sections. ${ }^{1}$

\section{METHOD}

Subjects were instructed to choose a variable rod so that its length was half way between the lengths of two given rods. On any trial, the two given rods were placed at prescribed positions on the table. The subject then selected one rod from a reference set that varied from 1 to $36 \mathrm{~cm}$ in $.5-\mathrm{cm}$ steps and placed it in line centrally between the two given rods. This selection process was repeated until the subject was satisfied. The experimenter then recorded the code number on the selected rod and replaced it and the two given rods with a new pair of given rods. All rods were 1/8-in.-diam tan wood dowel.

The instructions emphasized accuracy, and each session began with practice trials in which the three rods were arrayed vertically on the table, along the direction of gaze, aligned at their left ends, with the response rod inserted between the two given rods. This spatial configuration makes the task clear and facilitates accuracy because it requires bisection of position rather than of length. However, no feedback on accuracy was given at any time.

Each pair of given rods was listed on a separate index card. This deck of cards was shuffled between each replication to randomize order of presentation. Responses were recorded directly on these index cards.

\section{Experiment 1}

This experiment was actually the last in the series but is reported first for simplicity of exposition. Its main purpose was to check that long-term data from individual subjects would give the same data pattern as the earlier experiments.

The rods were in left-to-right linear array, with the variable rod placed centrally in the $50-\mathrm{cm}$ space that separated the proximal ends of the two given rods. The set of response rods lay in order on a cart to the right of the subject, slightly below the height of the table. The table was covered with brown paper, and the illumination was made as even as possible, since uneven illumination has been found to affect apparent length.

The left rod varied from 4 to $24 \mathrm{~cm}$ in $4-\mathrm{cm}$ steps. The right rod varied from 4 to $36 \mathrm{~cm}$ in $4-\mathrm{cm}$ steps but was never shorter than the left rod. This yielded 39 pairs in an incomplete factorial design whose pattern can be seen in Figure 1 below.

The subjects were six paid students. Each went through two replications on each of 5 days for a total of 10 replications per subject.

\section{Experiment 2}

This experiment was next to last in the series. It had two main purposes: to verify the pattern obtained in the earlier experiments and to check on possible effects of spatial location. General procedure was similar to that of Experiment 1.

Three spatial configurations were used in the main design. The three rods were in horizontal array, as in Experiment 1, but the response rod could be to the left of, in between, or to the right of the two given rods. The left rod varied from 4 to $20 \mathrm{~cm}$ in 4-cm steps, and the right rod varied from 4 to $36 \mathrm{~cm}$ in $4-\mathrm{cm}$ steps. However, the right rod was never less than the left rod, so there were 35 pairs in an incomplete factorial design similar to that of Experiment 1.

Supplementary data were also obtained for two other spatial 
configurations. In the vertical configuration, the three rods were spaced vertically with their left ends aligned. In the semivertical configuration, the two given rods were spaced similarly. The variable rod was placed between the two given rods in the vertical configuration, to the right of the lower given rod in the semivertical configuration. The upper rod was always $8 \mathrm{~cm}$, while the lower rod varied from 8 to $36 \mathrm{~cm}$ in 4-cm steps.

The subjects were 24 students, 8 for each of the three main spatial configurations. Each subject served for one replication of the 35 stimuli from the main configuration and for one replication of the 8 stimuli for each of the two final supplementary configurations.

\section{RESULTS}

\section{Bisection Patterning}

Experiment 1. In all the present experiments, the bisection responses exhibited the same odd pattern. This pattern is exhibited in Figure 1, which plots the results from Experiment 1 . The data points give the mean length of the bisecting rod as a function of the left and right bisectee rods.

The bottom data curve represents a left rod of $4 \mathrm{~cm}$ and a variable right rod. The line below it gives the physical mean which is nominally the correct value. The response rod is always longer than the physical mean; moreover, the difference increases as the right rod increases.

The top curve represents data for a left rod of $24 \mathrm{~cm}$ and a variable right rod, together with the

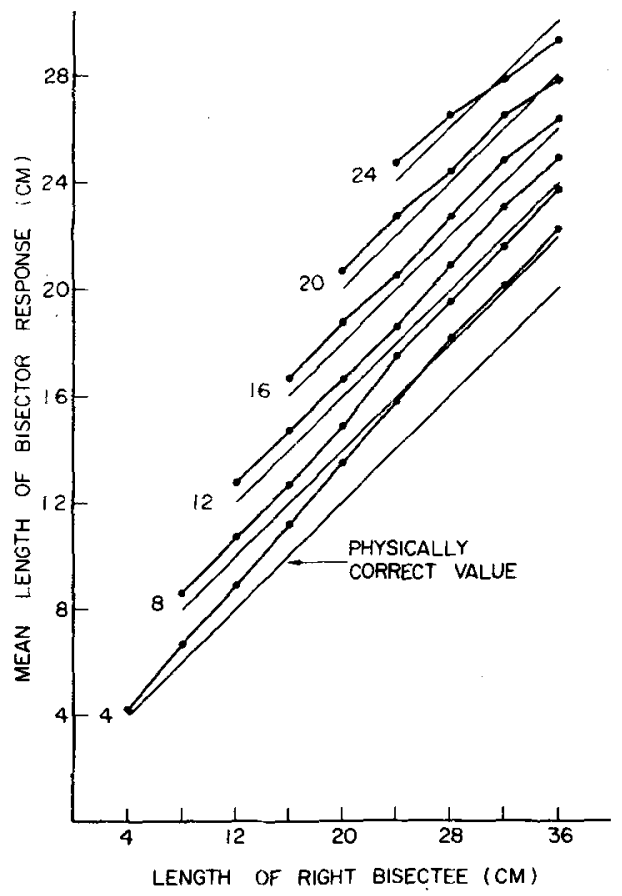

Figure 1. Mean length of bisector as a function of right bisectee (horizontal axis) and left bisectee (curve parameter). Straight line associated with each data curve gives the value of the physical arithmetic mean of the two bisectees. Data from Experiment 1. associated line of physical mean value. Again, the response is longer than the physical mean at the start of the curve. But, in this case, the difference decreases steadily and becomes negative as the response curve crosses under the physical mean.

This pattern can be simply summarized. When the left rod is short, the response curve lies above and his greater slope than the physical mean. But when the left rod is long, the response curve has smaller slope and falls below the physical mean. In equivalent terms, the physical mean looks too short when the left rod is short, too long when the left rod is long.

This pattern is very reliable. It was clear in the data of each individual subject in this experiment and in the group data from the other experiments. This pattern means that the data are inherently nonadditive and hence that the bisection model fails. The proof of this implication is deferred until the rest of the data are presented.

Experiment 2. The three panels of Figure 2 show the bisection data of Experiment 2. Each panel represents one spatial configuration according as the bisector rod (X) was placed to the left of, between, or to the right of the two given rods ( $L$ and $R$ ). The format is the same as for Figure 1 except in one respect; the higher curves are displaced successively leftward to increase clarity.

These data show the very same pattern as in Experiment 1. When the left rod is short, the response curve lies above the physical mean, and has greater slope. As the left rod increases in length, the slope of the response curve decreases steadily to become less than the slope of the physical mean. All three panels of Figure 2 show the same pattern. This pattern is not a peculiarity of one stimulus configuration.

The similarity of pattern for the three configurations of Figure 2 deserves emphasis, for it rules out one attentional interpretation of the nonadditivity. For any given $\mathrm{R}$ rod in the $\mathrm{L}-\mathrm{X}-\mathrm{R}$ configuration, the stimulus field expands as the $\mathrm{L}$ rod becomes longer. That could result in decreased attention to the $\mathrm{R}$ rod or to its distal part so that its effective length would be less for longer $L$ rods. Such an attentional effect, however, would produce different patterns of discrepancy for the L-R-X configuration, in which the $R$ rod is in the center of the stimulus field, and in the X-L-R configuration, in which it is farthest from the focus of response.

Further information on stimulus configuration comes from the supplementary data of Experiment 2 which are shown in the upper two rows of Table 1. The semivertical configuration shows the very same trend as in the comparable curves from Figure 2 (listed in the lower half of the table). The vertical configuration shows a nearly accurate response with a small, though uniform, constant error. Accuracy in the vertical configuration was expected, since here 


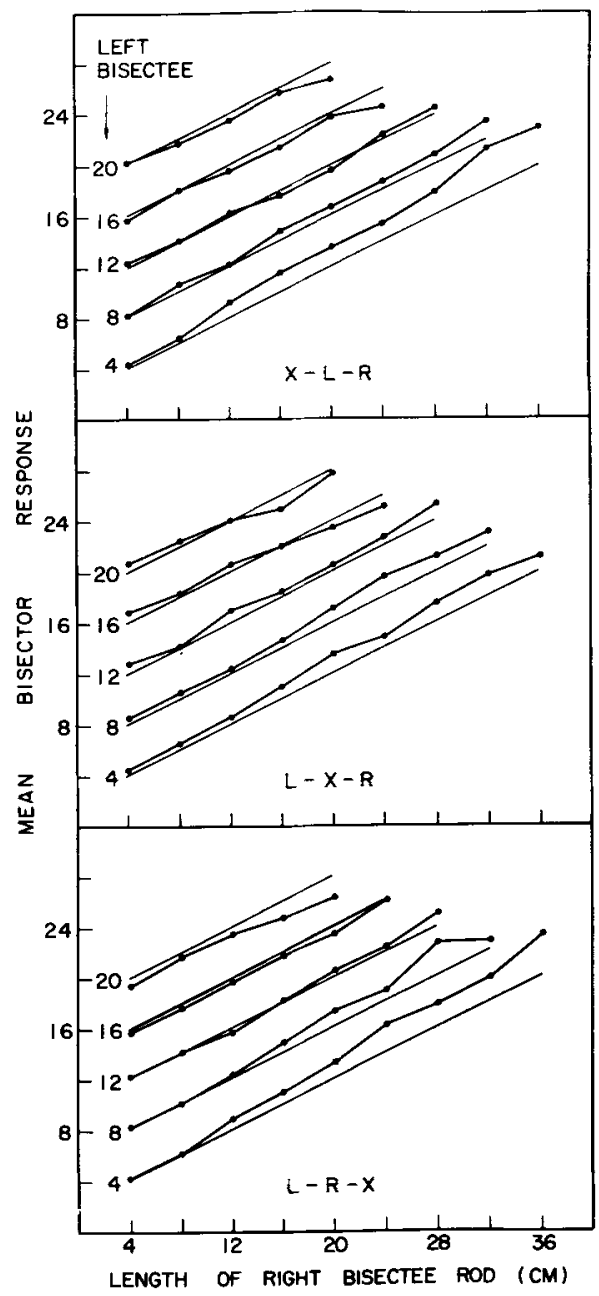

Figure 2. Mean length of bisector as a function of right bisectee (horizontal axis) and left bisectee (curve parameter). Straight line associated with each data curve gives the value of the physical arithmetic mean of the two bisectees. Each panel presents data for one spatial configuration of the three rods. Higher curves in each panel displaced successively leftwards for greater clarity. Data from Experiment 2.

the subject is effectively bisecting two positions rather than two lengths.

Preliminary experiments. Two preliminary experiments were also run. They showed the same pattern already noted and are mainly of interest for other checks on position effects. In particular, this preliminary work showed that left-right position effects were negligible, so this position factor was not balanced in the two subsequent experiments reported above.

In Experiment $A$, the $L$ rod ranged from 4 to $16 \mathrm{~cm}$ in $4-\mathrm{cm}$ steps. The $R$ rod ranged from 4 to $28 \mathrm{~cm}$ in 4-cm steps but was never shorter than the $\mathrm{L}$ rod. Each of 18 paid subjects served for four replications of these 22 rod combinations.

The horizontal L-X-R configuration was used, with the bisector between the two bisectees. $L$ and $R$ were counterbalanced across successive replications. Also, the vertical placement of the bisector varied on alternate replications, being either in line with the bisectees or $15 \mathrm{~cm}$ nearer the subject. No effect of either of these two spatial variables was found.

Experiment B was a small design to test whether white tagboard strips, $.5 \mathrm{~cm}$ wide, would give the same results as the wooden rods. In Part 1 , there were four successive replications for each of 12 subjects using the horizontal L-X-R configuration. The L strip was either 4 or $8 \mathrm{~cm}$, and the $R$ strip ranged from 4 to $20 \mathrm{~cm}$ in a $4-\mathrm{cm}$ steps. $L$ and $R$ were balanced across position on alternate replications, and they gave essentially the same results.

In Part 2 of Experiment $\mathbf{B}$, there were four replications using the semivertical configuration described in Experiment 2 above. Left and right now became top and bottom and were balanced over successive replications. In this semivertical configuration, the mean response was nearly veridical, in contrast to the results of Experiment 2 above. With this one possible exception, the results of these preliminary experiments agree with Experiment 2 above that spatial position has generally little effect for the stimuli used here.

\section{An Irreflexive Result}

Finally, a minor but curious aspect of the data is that the bisector of two equal lengths is slightly but systematically longer than the given lengths when it is located between them. This can be seen at the leftmost point of each curve of Figure 1 and in the center panel of Figure 2. In mathematical terms, this inequality is known as a violation of reflexivity

Table 1

Mean Length of Bisector of 8- and R-cm Rods, Experiment 2

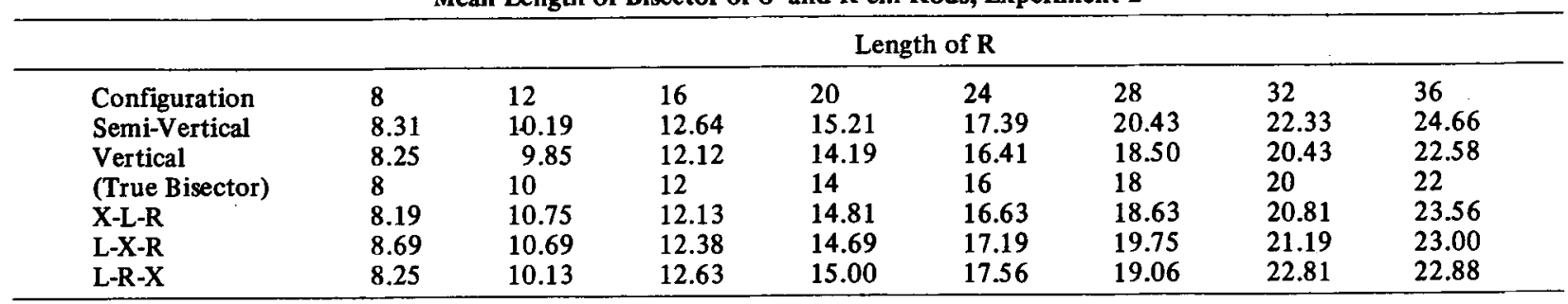


(Aczel, 1966). Reflexivity does appear to hold, however, when the bisector is to the left or right of the two given lengths. Post hoc test showed significantly greater inequality for the between location than for the two peripheral locations, $\mathrm{t}(22)=3.86, \mathrm{p}<.001$, while the inequality was not significantly different from zero for the two peripheral locations combined.

Reflexivity or lack thereof does not affect the basic pattern of the data noted above; that is the same in all three panels of Figure 2. Accordingly, the irreflexive result can be ignored in the analysis of the bisection model. It should be emphasized, however, that the bisection model can be generalized to allow for irreflexivity, as indeed is done in both the nonmetric analysis and the convexity analysis of footnote 2.

Empirically, the pattern of irreflexivity may not agree with other work (Piaget, 1969) which indicates that a line given greater attention tends to look longer. If that applied to the bisector, then it would be shorter than the two equal given lengths, not longer as was observed. It deserves mention, in any case, that the irreflexive trend was very uniform across subjects.

\section{Convexity Proof of Nonadditivity}

The convexity proof of nonadditivity is interesting because it provides a test solely in terms of the physical metric. This proof may thus be useful for tests of the bisection model on other stimulus dimensions.

The essential assumption of the convexity proof is that the psychophysical function $V$ is convex, either up or down. Since spatial order effects were negligible in the present data, it will be assumed that $\mathrm{w}_{1}=\mathrm{w}_{2}$ in the bisection model of Equations 3-4. Taken together, convexity and the bisection model imply that the data pattern of Figure 1 is impossible. The convexity assumption, which includes the log and power functions as special cases, hardly seems disputable. It must follow, then, that the averaging model for bisection is invalid. The proof goes as follows.

A function or curve is said to be convex when the chord between any two points on the curve lies entirely on one side of the curve itself. Figure 3 shows a $\mathrm{V}$ function that is convex down. Inspection of the figure shows that the $\mathrm{V}$ value of the mean of stimuli $S_{1}$ and $S_{2}$ is greater than the mean of their separate $\mathrm{V}$ values:

$$
\mathrm{V}\left[\left(\mathrm{S}_{1}+\mathrm{S}_{2}\right) / 2\right]>\left[\mathrm{V}\left(\mathrm{S}_{1}\right)+\mathrm{V}\left(\mathrm{S}_{2}\right)\right] / 2 .
$$

This inequality holds for any two stimuli by virtue of convexity, for the term on the left lies on the curve whereas the term on the right lies directly below at the midpoint of the chord connecting $V\left(S_{1}\right)$ and $\mathrm{V}\left(\mathrm{S}_{2}\right)$.
The $\mathrm{V}^{-1}$ transform is now taken of both terms of Inequality 5 . This preserves the inequality because $\mathrm{V}^{-1}$ is monotone increasing. Since $\mathrm{V}^{-1} \mathrm{~V}$ is the identity function, the term on the left becomes

$$
\mathrm{V}^{-1}\left\{\mathrm{~V}\left[\left(\mathrm{~S}_{1}+\mathrm{S}_{2}\right) / 2\right]\right\}=\left(\mathrm{S}_{1}+\mathrm{S}_{2}\right) / 2 .
$$

The term on the right becomes the response predicted by the bisection model:

$$
\mathrm{V}^{-1}\left\{\left[\mathrm{~V}\left(\mathrm{~S}_{1}\right)+\mathrm{V}\left(\mathrm{S}_{2}\right)\right] / 2\right\}=\mathrm{R} .
$$

This follows from Equation 4 above, with $w_{1}=w_{2}$ $=1 / 2$.

If $\mathrm{V}$ is convex down, therefore, Equations 5, 6, and 7 imply that the mean physical value of two stimuli is greater than the physical value of their bisector:

$$
\left(\mathrm{S}_{1}+\mathrm{S}_{2}\right) / 2>\mathrm{R} .
$$

By symmetry, the opposite inequality must hold if $\mathrm{V}$ is convex up:

$$
\left(\mathrm{S}_{1}+\mathrm{S}_{2}\right) / 2<\mathrm{R} .
$$

It follows, therefore, that if the observed bisector is physically smaller (larger) than the physical mean for any one pair of bisectee stimuli, then the same must be true for every other pair. This contradicts the data of Figure 1 which show both directions of inequality. Accordingly, the bisection model of Equation 3-4 must be rejected for these data.

This convexity analysis can be extended to allow for order effects, that is, $w_{1} \neq w_{2}$. The above reasoning may be applied directly, noting that the weights

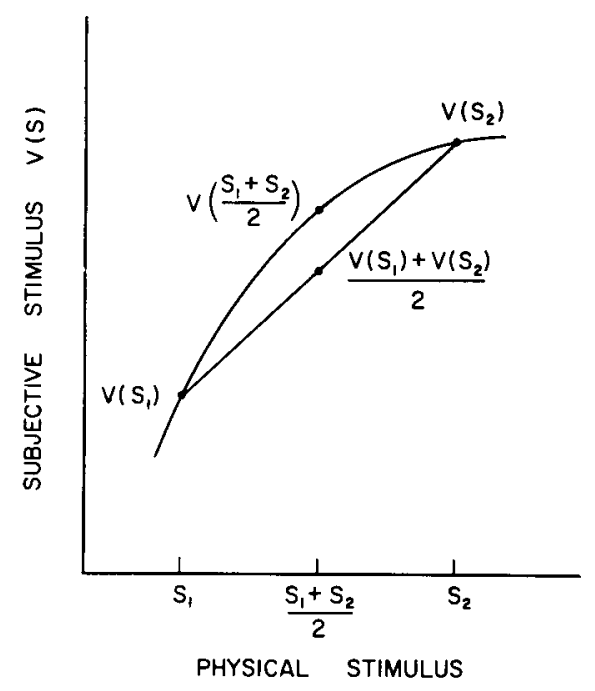

Figure 3. Convex down function illustrating that the sensation value of the mean stimulus is greater than the mean of the separate sensation values. 
drop out when the subjective response is averaged over the two orders of presentation. This analysis leads to the following result: If the bisector is smaller (larger) than the physical mean of the bisectees for both orders, then the psychophysical function must be convex down (up) and the bisector cannot be larger (smaller) than the physical mean of the bisectees for both orders of presentation for any other pair of stimuli.

This last result may not handle all cases, especially when the instructions call for unequal sections. If the two weights are sufficiently different, the bisector can be larger for one order of presentation, smaller for the other, regardless of the shape of the psychophysical function. ${ }^{2}$

\section{Nonmetric Proof of Nonadditivity}

The nonmetric analysis has two points of interest beyond the present data. First, it illustrates an error theory for nonmetric analysis recently developed in functional measurement. Second, it provides an empirical case in which the data from even a relatively small design resist transformation to additivity.

If the bisection model is valid, then the observed responses can be monotonically transformed to additivity. For this purpose, the ADDALS program of deLeeuw, Young, and Takane (1976) was applied. This program computes the monotone transformation that makes the data as additive as possible.

This transformation was applied separately to the data of each subject in Experiment 1. The mean transformed data are plotted in the left panel of
Figure 4. If the transformation has been successful, then these curves should be parallel.

Although these curves may look reasonably parallel, that is an optical illusion. Direct measurement of the vertical spread shows a substantial convergence from left to right, similar to that in the raw data. For the complete 4 by 6 factorial subdesign in the lower right, for example, the vertical spread decreases by a bit more than $25 \%$ from left to right. It would seem, therefore, that no monotone transformation can eliminate the convergence in the raw data. However, this conclusion requires a statistical test to check that the observed nonparallelism is reliable.

Unfortunately, as is well known, analysis of variance is not generally applicable to monotonically transformed data. There are two difficulties. First, the transformation uses up an unknown number of degrees of freedom. Second, the transformed data are all intercorrelated through their dependence on a common transformation function.

Fortunately, these difficulties can be avoided by making separate transformations for each subject (or for each replication for an individual subject). The transformed data are then independent across subjects (or replications). And although they are intercorrelated within subjects (or replications), the repeated-measurements analysis of variance allows for that (Anderson, 1975, p. 478, 1977). For present convenience, this analysis was restricted to the complete 4 by 6 factorial subdesign in the lower right section of the left panel of Figure 4. Parallelism

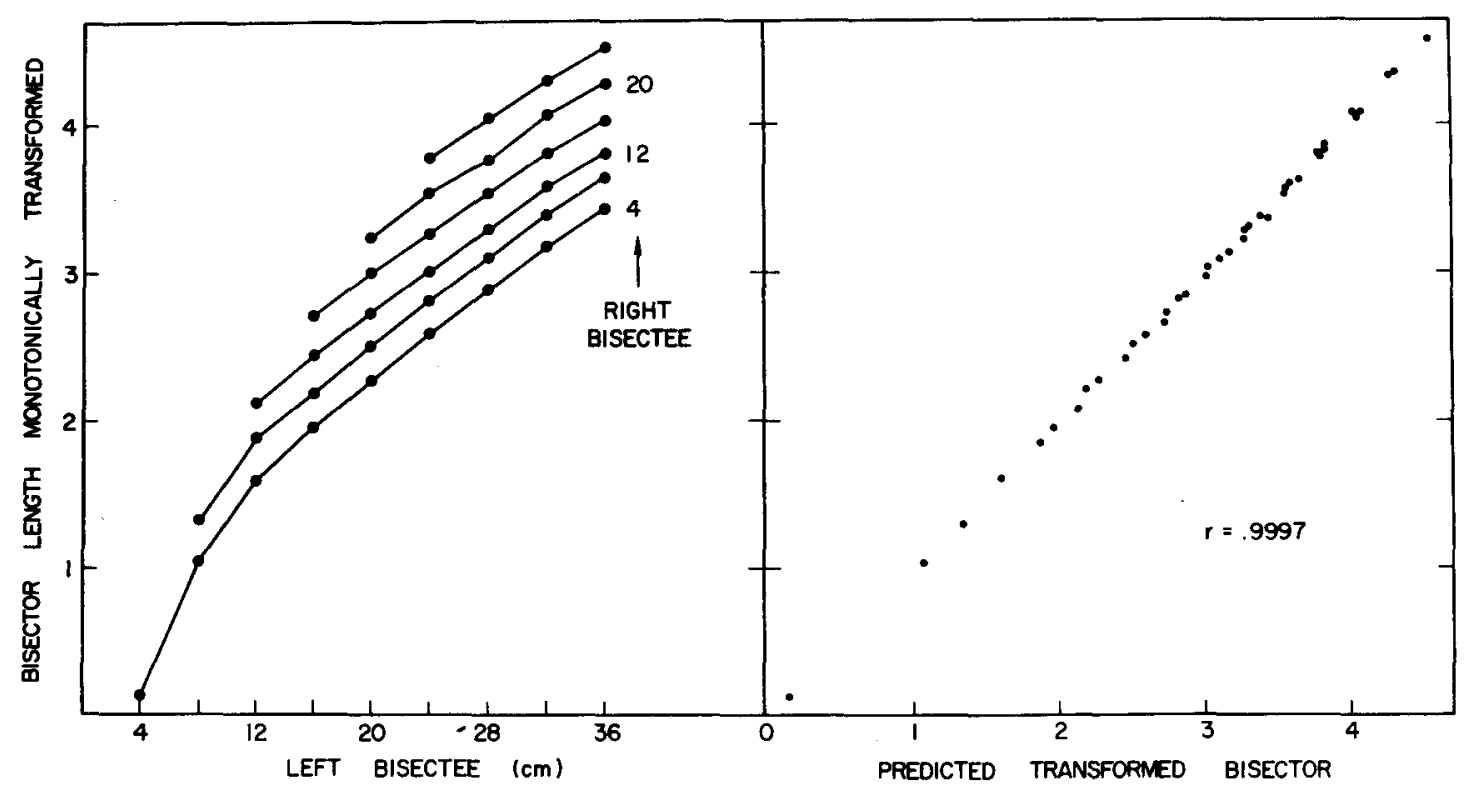

Figure 4. Left panel shows bisection data of Experiment 1 monotonically transformed to be as additive as possible. Curves converge rightward showing that the data are truly nonadditive. Right panel shows predictions obtained from fitting additive model to the nonadditive data of the left panel. 
corresponds to zero interaction term in the analysis. Since the interaction was significant, $F(15,75)$ $=2.94, \mathrm{p}=.001$, the transformed data are reliably nonparallel. It follows that the observed bisection data are inherently nonadditive, a conclusion that agrees that the above convexity analysis.

This nonmetric analysis, it may be noted, actually tests a more general model than the bisection model of Equation 4. The only property at issue in the test is additivity. There is no restriction on the weights, therefore, and an additive constant is admissible. This more general model is equivalent to that obtainable from Pfanzagl's (1968) bisymmetry axiom (see Krantz, Luce, Suppes, \& Tversky, 1971, p. 295).

\section{Need for Test of Goodness of Fit}

The neccessity for a proper statistical test is underscored by the correlation-scatterplot analysis in the right panel of Figure 4. Although the data of the left panel are reliably nonadditive as already shown, an additive model was fit using least squares. The right panel of Figure 4 shows the scatterplot of the data points in the left panel as a function of the values predicted by the additive model. Deviations from the diagonal line of perfect fit are miniscule. The correlation is surpassingly high, $r=.9997$. Judged by these correlation-scatterplot statistics, it would seem that the additive model had done very well. But these correlation-scatterplot statistics are deceiving; they seem to test the model but they do not really do so. This example is but one of many that tell the same story (Anderson, 1976a, 1977; Anderson \& Shanteau, 1977; Birnbaum, 1973).

\section{DISCUSSION}

\section{The Bisection Model}

Despite its seeming simplicity, and despite a century of attention, the bisection model has proved elusive (Weiss, 1973, 1975). The previous work on grayness (Anderson, 1976b; Weiss, 1975) apparently provides the first clear demonstration of a success of the model. This conclusion was supported both by tests of goodness of fit and by cross-task validation.

The revised mel scale for pitch (Stevens \& Volkman, 1940), perhaps the most prominent example of the use of bisection-type methods, rests on the arbitrary assumption that the model is correct. The computational procedure will always yield a presumptive scale, but lacks a test of goodness of fit (Torgerson, 1961; Weiss, 1973). Torgerson attempted to provide a test by looking for a linear relation between scales obtained from overlapping segments on the stimulus scale. However, such linearity can result merely from absence of order effects, and that is not sufficient to establish the model as the present results demonstrate. Of course, the methods of bisection and equisection may well yield valid scales of pitch. However, the validity of such scales rests completely on the validity of the model, and that is not yet known.

On the other hand, the present experiments on length apparently provide the first clear demonstration of a failure of the bisection model. Some previous investigators have reported difficulties, but only for special cases. Thus, the so-called hysteresis effects (e.g., Gage, 1934) were once considered to infirm the model. However, Gage only considered the case of equal weights, $w_{1}=w_{2}$ in Equation 4 . It is now generally recognized that hysteresis and other kinds of order effects can, at least in principle, be handled in terms of unequal weight parameters.

Aside from the functional measurement analyses of grayness (Anderson, 1976b; Weiss, 1975), only a few studies have allowed for unequal weights, and none of them seems to reach a clear conclusion. Perhaps the most careful and thorough study is that of Fagot and Stewart (1970) on bisection of brightness. They first showed that a model with equal weights did not fit their data. To allow unequal weights, however, they found it necessary to assume a specific form for the psychophysical function. This latter analysis still appeared to show some modeldata inconsistencies which, as they note, could result from an incorrect psychophysical function, an incorrect model, or both.

Adams and Fagot (1975) attempted to bypass the unequal weighting problem by adding left and right inverse judgments. (If $x<y$ are the given stimuli, then the left inverse is the response such that $\mathrm{x}$ bisects it and y.) The present functional measurement approach accomplishes the same goal more simply, for the additional judgments are not necessary and a proper error theory is available.

\section{Interpretation of the Discrepancies}

The cause of the present failure of the bisection model is unknown. However, some relevant considerations may be noted briefly.

If only scaling were at issue, then the discrepancies would perhaps not seem overly serious. If the bisection equation is approximately correct, then it can yield approximate scales. A mild bias might be tolerable, for its cause would not be relevant.

However, if the bisection process itself has primary concern, then the evaluation of the discrepancies is more problematical. Significant discrepancies need not require rejection of a model (Anderson, 1977), for they could reflect the approximate character of some simplifying assumption. On the other hand, they could also signal inadequacies in the basic assumptions.

In the present case, a provisional judgment is that the discrepancies are serious. Even when monotone response transformation is allowed, the data still exhibit moderate nonparallelism, of the same pattern 
as in the raw data. Further, the response and the physical mean did not even have a constant ordinal relation. The physical mean looked too short when the left rod was short, too long when the left rod was long.

A straightforward generalization of the bisection model would allow for differential weighting. In the averaging form of Equation 3, the weights would depend on stimulus value. Such differential weighting has been demonstrated for symbolic stimuli (Anderson, 1974b), which suggests the same possibility for perceptual stimuli. In fact, this differential weight averaging model implies the observed convergence pattern under the plausible assumption that longer lengths get greater weight. Moreover, this model is inherently nonadditive and so could account for the failure of additivity. Closer analysis is less promising. If $\mathrm{V}$ is convex down, the model can be eliminated by a direct extension of the proof given in Footnote 2. The case with $\mathrm{V}$ convex up has not been ruled out, but several special cases based on power function approximations have not been able to account for the slope properties of the observed error function.

An alternative hypothesis is that the subjects are equating sense ratios rather than sense distances (Rose \& Birnbaum, 1975; Torgerson, 1961). Analogous to Equations 2 and 3, the ratio formulation for bisection would be (Anderson, 1970, p. 159):

$$
\mathrm{r} / \mathrm{s}_{1}=\mathrm{w}\left(\mathrm{s}_{2} / \mathrm{r}\right) \text {, or } \mathrm{r}^{2}=\mathrm{ws}_{1} \mathrm{~s}_{2} \text {. }
$$

But this model, essentially a subjective geometric mean, is monotonically equivalent to the original model because $\log r^{2}$ is additive. Further, a linear composite of these two models is also monotonically equivalent to the original model (Anderson, 1970, Equation 18). Since both the convexity and the nonmetric analyses allow for monotone transformation, the discrepancies from the model in Figure 1 cannot be attributed to such a ratio judgment, either alone or combined with a difference judgment.

A different possibility is that some geometrical illusion is present, overlaid on a basic bisection model. Thus, the bisectee rods could exert assimilation and contrast effects on the bisector. However, the results of Clavadetscher and Anderson (1977) suggest that assimilation-contrast effects would be small at the present interrod distances. Further, the same pattern of discrepancy appears when the bisector is placed to the left, right, or in between the bisectees (Figure 2).

Stevens (1971) has argued that bisection induces a partition bias that yields invalid scales on prothetic continua. However, his argument does not seem to be correct since the bisection model has been successful in two studies of grayness, as already noted. The resulting functional grayness scales were radically different from the magnitude estimation scale, it is true, but these functional scales were validated by both internal consistency and by cross-task consistency. On at least one prothetic continuum, therefore, the bisection model does appear to be valid.

Of course, the failure of the bisection model may result merely from some peculiarity of the present experimental conditions. Some objections of this kind have been discussed above, but others remain. Even if the result is not general, however, it still presents an interesting puzzle in length perception.

\section{Measurement Theory}

The problem of bisection illustrates the difference between the functional and axiomatic approaches to psychological measurement (Anderson, 1970, 1974a; Cliff, 1973 , p. 480). Accordingly, the functional measurement approach to bisection will be compared with the axiomatic approach adopted by Pfanzagl (1968) and in conjoint measurement (Krantz, Luce, Suppes, \& Tversky, 1971).

The functional approach views bisection as a task of stimuius integration. The commonsense model of equated sense distance is taken as the starting point. The focus is on the perceptions that operate in the bisection process itself, and the evaluation of the simple bisection model is only one step in the process analysis. When the model can be established, as it was for grayness, the main gain is knowledge about bisection processes. At the same time, of course, the model also provides a frame for scaling and for determining the psychophysical law. Failure of the model, as for length, disallows the scaling but may nevertheless shed light on the bisection processes.

The axiomatic approach has a different orientation, for it is not grounded in process assumptions. It seeks a set of axioms that will mathematically imply an additive model. However, these axioms are not concerned with the assumption of equated sense distances, or with any analogous psychological process. This point is well illustrated by certain key axioms, those that deal with order relations among observable responses.

In Pfanzagl's formulation, the key axiom is the bisymmetry axiom. Applied to bisection, it states that one particular sequence of three bisections leads to the same result as another particular sequence of three bisections. ${ }^{3}$ This axiom clearly does not represent the psychological processes involved in any one bisection.

The same point applies to the key axiom of double cancellation in conjoint measurement (Krantz et al., 1971, Section 6.9.1). It involves comparisons among responses on six different trials. But these comparisons are made by the investigator, not by the sub- 
ject. This axiom thus has no referent to the psychological processes involved in any one bisection.

The axioms of abstract measurement theory are thus quite different from the assumptions of Thurstonian scaling and signal detection theory, say, or from the assumption that bisection involves an equating of sense distances. These latter axioms refer to psychological processes that are thought to underlie a given response. In contrast, the axioms of bisymmetry and double cancellation do not refer to psychological processes, but merely to the relative sizes of overt responses on different trials.

If the axioms of abstract measurement theory do not deal with the functional processes, what value can they have? One obvious answer is that they can provide a basis for testing the model. The axiomatic approach has generally made it a principle that only ordinal data and ordinal tests are allowable. The axioms provide a potential basis for testing goodness of fit using only ordinal information. This point is well illustrated by the fact that the axiom of double cancellation can be viewed as an ordinal implication of parallelism or additivity in two linked 2 by 2 designs (Krantz et al., 1971, p. 250). In this respect therefore, abstract measurement theory has a role that is directly analogous to the role of monotone transformation and analysis of variance in functional measurement.

Does the axiomatic approach have any deeper value or significance than that of providing a basis for model analysis with ordinal data? There seems to be some feeling that it does, but the rationale remains obscure. Since the axioms do not refer to psychological processes, they do not constitute a psychological theory in the usual sense. That the model can be derived from such ordinal axioms is an impressive mathematical exercise, but confers no scientific cachet on the model. In the writer's view, therefore, the axiomatic approach to measurement theory has no deeper substantive or epistemological role than the statistical techniques used in functional measurement.

In actual use, as is well known, the axiomatic approach is weak. The theorems assume error-free data and are ill-suited to handle real data. As a consequence, empirical applications have been extremely rare (e.g., Cliff, 1973, p. 478; Falmagne, 1976, p. 65; Tukey, 1969, p. 88).

Functional measurement, in contrast, has had many empirical applications. One reason is that metric response measures are allowable and have been found to be quite useful. Ordinal analysis is also necessary (Anderson, 1962) and has become practicable through the development of the error theory illustrated above. This same approach may be applied to many other algebraic models in psychology, including nonlinear or interactive processes that seem generally beyond the reach of the axiomatic approach.

Functional measurement owes much to the work of Kruskal (1965), de Leeuw et al. (1976), and Young, de Leeuw, and Takane (1976) on additivity analysis. Their work has provided a vital tool for the study of bisection and for the general error theory for ordinal data outlined above.

Young et al. (1976) contrast three schools of thought about additivity: optimal scaling, as represented by their own work, traditional analysis of variance, and conjoint measurement. Functional measurement represents a fourth school of thought. It makes important use of the tools of analysis of variance and of optimal scaling, as has been illustrated above. However, its central concern is not with mathematics or statistics but with the psychological processes that give rise to the model, be they additive or nonadditive. In this regard (Anderson, 1970 , p. $168 ; 1974$ a, p. $291 ; 1975$, p. 480), the present approach is part of a long tradition about the nature of measurement in psychology.

\section{REFERENCES}

ACZEL, J. Lectures on functional equations and their applications. New York: Academic Press, 1966.

ADAMs, E. W., \& FAGOT, R. F. On the theory of biased bisection operators and their inverses. Joumal of Mathematical Psychology, 1975, 12, 35-52.

ANDERSON, N. H. On the quantification of Miller's conflict theory. Psychological Review, 1962, 69, 400-414.

ANDERSon, N. H. Functional measurement and psychophysical judgment. Psychological Review, 1970, 77, 153-170.

ANderson, N. H. Algebraic models in perception. In E. C. Carterette \& M. P. Friedman (Eds.), Handbook of perception (Vol. 2). New York: Academic Press, 1974. (a)

ANDERSON, N. H. Information integration theory: A brief survey. In D. H. Krantz, R. C. Atkinson, R. D. Luce, \& P. Suppes (Eds.), Contemporary developments in mathematical psychology (Vol. 2). San Francisco: Freeman, 1974. (b)

ANDERson, N. H. On the role of context effects in psychophysical judgment. Psychological Review, 1975, 82, 462-482.

ANDERSON, N. H. How functional measurement can yield validated interval scales of mental quantities. Journal of Applied Psychology, 1976, 61, 677-692. (a)

ANDERSON, N. H. Integration theory, functional measurement, and the psychophysical law. In H.-G. Geissler \& Yu. M. Zabrodin (Eds.), Advances in psychophysics. Berlin: VEB Deutscher Verlag, 1976. (b)

Anderson, N. H. Note on functional measurement and data analysis. Perception \& Psychophysics, 1977, 21, 201-215.

Anderson, N. H., \& Shanteau, J. Weak inference with linear models. Psychological Bulletin, 1977, in press.

Birnbaum, M. H. The devil rides again: Correlation as an index of fit. Psychological Bulletin, 1973, 79, 239-242.

Bogartz, R. S., \& WACKWITZ, J. H. Polynomial response scaling and functional measurement. Journal of Mathematical Psychology, 1971, 8, 418.443.

Clavadetscher, J. E., \& Anderson, N. H. Comparative judgment: Tests of two theories using the Baldwin figure. Journal of Experimental Psychology: Human Perception and Performance, 1977, 3, 119-133.

Cliff, N. Scaling. Annual Review of Psychology, 1973, 24, 473-506. 
DE Leeuw, J., Young, F. W., \& TAkane, Y. Additive structure in qualitative data: An alternating least squares method with optimal scaling features. Psychometrika, 1976, 41, 471-503.

FAGOT, R. F. A model for equisection scaling. Behavioral Science, 1961, 2, 127-133.

FAGOT, R. F., \& STEwART, M. R. Test of a response bias model of bisection. Perception \& Psychophysics, 1970, 7, 257-262.

FAlmagne, J.-C. Random conjoint measurement and loudness summation. Psychological Review, 1976, 83, 65-79.

GAGE, F. H. An experimental investigation of the measurability of auditory sensation. Proceedings of the Royal Society [London], 1934, 116B, 103-122.

Krantz, D. H., Luce, R. D., Suppes, P., \& Tversky, A. Foundations of measurement (Vol. 1). New York: Academic Press, 1971.

Kruskal, J. B. Analysis of factorial experiments by estimating monotone transformations of the data. Journal of the Royal Statistical Society $[B], 1965,27,251-263$.

MarKs, L. E. Sensory processes. New York: Academic Press, 1974.

Pfanzagl, J. Theory of measurement. New York: Wiley, 1968.

Puaget, J. The mechanisms of perception. New York: Basic Books, 1969.

Rose, B. J., \& Birnbaum, M. H. Judgments of differences and ratios of numerals. Perception \& Psychophysics, 1975, 18, 194-200.

Stevens, S. S. Issues in psychophysical measurement. Psychological Review, 1971, 78, 426-450.

Stevens, S. S., \& Volkman, J. The relation of pitch to frequency: A revised scale. American Journal of Psychology, 1940, 53, 329-353.

ToRgerson, W. S. Distances and ratios in psychological scaling. Acta Psychologica, 1961, 19, 201-205.

TukeY, J. W. Analyzing data: Sanctification or detective work? American Psychologist, 1969, 24, 83-91.

WEISS, D. J. A functional measurement analysis of equisection. Unpublished PhD dissertation, University of California, San Diego, 1973.

WEISS, D. J. Quantifying private events: A functional measurement analysis of equisection. Perception \& Psychophysics, 1975, 17, 351-357.

WeIss, D. J., \& ANDERson, N. H. Use of rank order data in functional measurement. Psychological Bulletin, 1972, 78, 64-69.

Young, F. W., De Leeuw, J., \& TAKane, Y. Regression with qualitative and quantitative variables: An alternating, least squares method with optimal scaling features. Psychometrika, $1976,41,505-529$.

\section{NOTES}

1. Multisection judgments have theoretical interest since they allow monotone discrimination between difference and ratio judgments (Anderson, 1970, pp. 159-160). It should be added that the multisection model did poorly in what seems to be the only article that provided a test of goodness of fit (Weiss, 1975).

2. A more general, though more complex, convexity proof can be obtained in terms of the slope of the "error" function,

$$
E\left(S_{1}, S_{2}\right)=R\left(S_{1}, S_{2}\right)-\left(S_{1}+S_{2}\right) / 2,
$$

which is the difference between the physical value of the bisection response and the physical mean value. This proof allows for the general bisection model with unequal weights and an additive constant, c. Analogous to Equation 4, the general model may be written

$$
R\left(S_{1}, S_{2}\right)=V^{-1}\left[w_{1} V\left(S_{1}\right)+w_{2} V\left(S_{2}\right)+c\right] .
$$

The error function then becomes,
$E\left(S_{1}, S_{2}\right)=V^{-1}\left[w_{1} V\left(S_{1}\right)+w_{2} V\left(S_{2}\right)+c\right]-\left(S_{1}+S_{2}\right) / 2$.

Three cases need to be considered, according as $\mathrm{V}$ is linear, convex down, or convex up. For convenience, it is assumed that $S_{1}<S_{2}$.

$V$ is linear. If $\mathrm{V}=\mathrm{aS}+\mathrm{b}$, then

$$
\mathrm{V}^{-1}\left[\mathrm{w}_{1} \mathrm{~V}\left(\mathrm{~S}_{1}\right)+\mathrm{w}_{2} \mathrm{~V}\left(\mathrm{~S}_{2}\right)+\mathrm{c}\right]=\mathrm{w}_{1} \mathrm{~S}_{1}+\mathrm{w}_{2} \mathrm{~S}_{2}+\mathrm{c} / \mathrm{a} \text {. }
$$

Hence the error function becomes

$$
E\left(S_{1}, S_{2}\right)=\left(w_{1}-.5\right) S_{1}+\left(w_{2}-.5\right) S_{2}+c / a .
$$

Differentiation of the error function with respect to $S_{2}$ shows that the slope, $\left(w_{2}-.5\right)$, is constant. This disagrees with the data of Figure 1 which show positive slope for $S_{1}$ small and negative slope for $\mathrm{S}_{1}$ large.

$V$ is convex down. Differentiate Equation 11 with respect to $\mathrm{S}_{2}$ to get the slope of the error function as a function of $\mathrm{S}_{2}$ :

$E^{\prime}\left(S_{1}, S_{2}\right)=V^{-1}{ }^{\prime}\left[w_{1} V\left(S_{1}\right)+w_{2} V\left(S_{2}\right)+c\right] w_{2} V^{\prime}\left(S_{2}\right)-1 / 2$.

Since $\mathrm{V}$ is convex down, $\mathrm{V}^{-1}$ is convex up, and its derivative, $\mathrm{V}^{-1^{\prime}}$, is an increasing function of its argument. Also, $\mathrm{V}^{\prime}$ is positive, since $V$ is monotone increasing. For fixed $S_{2}$, therefore, the bisection model implies that the slope of the error function is an increasing function of $S_{1}$. That is contrary to the data of Figure 1 which show that the slope of the response function, and equivalently of the error function, decreases steadily as the length of the left $\mathrm{S}_{1}$ rod increases.

$V$ is convex up. Since the relative sizes of the weights can be determined empirically, the stimulus variables can be labeled so that $w_{1}<.5<w_{2}$. It is assumed that $c$ is small enough that

$$
w_{1} V\left(S_{1}\right)+w_{2} V\left(S_{2}\right)+c<V\left(S_{2}\right)
$$

Since $\mathrm{V}$ is convex up, $\mathrm{V}^{-1}$ is convex down, and its derivative, $\mathrm{V}^{-\mathbf{1}^{\prime}}$, is a decreasing function of its argument. Accordingly, application of the $\mathrm{V}^{-1}$-transform to the above inequality yields

$$
V^{-1}\left[w_{1} V\left(S_{1}\right)+w_{2} V\left(S_{2}\right)+c\right]>V^{-1}\left[V\left(S_{2}\right)\right]
$$

This inequality and Equation 12 imply

$$
E^{\prime}\left(S_{1}, S_{2}\right)>\left\{V^{-1}\left\{V\left(S_{2}\right)\right] V^{\prime}\left(S_{2}\right)\right\} w_{2}-1 / 2 .
$$

But the expression in braces equals unity by the rule for the derivative of an inverse function. In this case, therefore, the slope of the error function is always positive. That is contrary to the data of Figure 1 which show negative slopes for $S_{1}$ large.

3. Given four stimuli, denoted by $S_{1}, S_{2}, S_{3}$ and $S_{4}$, the bisymmetry axiom states that

$$
\text { bisector [bisector }\left(\mathrm{S}_{1}, \mathrm{~S}_{2}\right) \text {, bisector }\left(\mathrm{S}_{3}, \mathrm{~S}_{4}\right) \text { ] }
$$

$$
=\text { bisector }\left[\text { bisector }\left(S_{1}, S_{3}\right) \text {, bisector }\left(S_{2}, S_{4}\right)\right] \text {. }
$$

4. Allowance for monotone transformation is, of course, an essential part of functional measurement (Anderson, 1962, p. 408; Weiss \& Anderson, 1972). However, the original suggestion for a power series transformation, although it seemed to lead to a better error theory (Bogartz \& Wackwitz, 1971; Weiss, 1975), has been less useful in practice than the MONANOVA and ADDALS programs developed by Kruskal (1965) and by de Leeuw et al. (1976), respectively. It may be added that the method for testing goodness of fit outlined in the text should be applicable in multidimensional scaling which has lacked an error theory.

(Received for publication October 1, 1976; revision accepted June 13, 1977.) 